\title{
Examination of the Reduced Affinity of the Thymidylate Synthase G52S Mutation for FdUMP by Ab Initio and Semi-empirical Studies
}

\author{
Anne-Marie Sapse, ${ }^{1}$ Gina M. Capiaux ${ }^{2,3}$ and Joseph R. Bertino ${ }^{2,3}$ \\ ${ }^{1}$ City University of New York Graduate School and John Jay College, New York, New York, and Rockefeller \\ University, New York, New York, USA \\ ${ }^{2}$ Program of Molecular Pharmacology and Therapeutics, Memorial Sloan-Kettering Cancer Center, \\ New York, New York, USA \\ ${ }^{3}$ Graduate School of Medical Sciences, Cornell University, New York, New York, USA \\ Accepted [add date]
}

\begin{abstract}
Background: The G52S mutation in the $\mathrm{Arg}^{50}$ loop of thymidylate synthase leads to decreased binding of FdUMP. It has been suggested that the mutation affects the $\mathrm{Arg}^{50}$ residue (within the $\mathrm{Arg}^{50}$ loop) responsible for binding the phosphate of FdUMP. The binding of the methylguanidinium moiety as a model for $\operatorname{Arg}^{50}$ to a methylphosphate entity as a model for FdUMP was investigated with theoretical calculations, as well as the structure of the $\mathrm{Arg}^{50}-\mathrm{Thr}^{51}-\mathrm{Gly}^{52}$ tripeptide in comparison with the $\mathrm{Arg}^{50}-\mathrm{Thr}^{51}-\mathrm{Ser}^{52}$ tripeptide.

Methods: Gaussian-98 and PC Spartan programs were used to perform Hartree-Fock and Post-Hartree-Fock

quantum chemical calculations as well as MNDO (semiempirical calculations).

Results: It was found that the strongest binding occurs between the negative methylphosphate ion and methylguanidine. The replacement of $\mathrm{Gly}^{52}$ by $\mathrm{Ser}^{52}$ leads to a significant displacement of $\mathrm{Arg}^{50}$, which may be responsible for the decreased binding to FdUMP.

Conclusion: The arginine-phosphate binding appears to be geometry dependent. Thus, the displacement of the $\mathrm{Arg}^{50}$ residue, as observed in these calculated models, upon mutation of $\mathrm{Gly}^{52}$ to Ser may contribute to decreased binding of FdUMP to mTS (G52S).
\end{abstract}

\section{Introduction}

Thymidylate synthase (TS) is an essential enzyme in proliferating cells and an important target for a variety of anticancer drugs. This enzyme is responsible for catalyzing the de novo biosynthesis of thymidylate, which is necessary for DNA synthesis and repair. The mechanism involves the reductive transfer of a methylene group from the cofactor, 5, 10-methylene-tetrahydrofolate $\left(\mathrm{CH}_{2} \mathrm{H}_{4}\right.$ folate), to the 5 position of the substrate, $2^{\prime}$-deoxyuridine $5^{\prime}$-monophosphate (dUMP), to form 2'-deoxythymidine 5'-monophosphate (dTMP).

Many TS analogs of both the substrate, dUMP, and the cofactor, $\mathrm{CH}_{2} \mathrm{H}_{4}$ folate, have been synthesized and investigated as potential anticancer therapeutics. Two clinically useful substrate analogs are 5-fluorouracil (5-FU) and fluorodeoxyuridine (FdUrd), which are metabolized in vivo to 5-fluoro2-deoxyuridylate (FdUMP). FdUMP acts as an inhibitor of TS by forming a stable ternary complex with TS and the folate cofactor, resulting in inhibition of enzyme function. In addition to these sub-

Address correspondence and reprint request to: Anne-Marie Sapse, PhD, John Jay College, 445 W. 59th Street, New York, NY 1019. Phone: 212-588-0487; Fax: 212-935-359. strate analogs, novel folate cofactor analogs, such as raltitrexed (Tomudex) and thymitaq have also been designed as specific inhibitors of TS activity (la).

To better understand the mechanism of TS enzyme catalysis, several laboratories have correlated TS enzyme structure and activity using mutagenesis. Following substitution of different amino acids at random or specific points within the enzyme, enzyme activity and affinity for inhibitors are measured to determine how a particular residue(s) contributes to the overall mechanism. Recently, several mutations at highly conserved active site residues have been made for human TS (hTS) $\left(\mathrm{Ile}^{108}\right.$, $\mathrm{Leu}^{221}$, and $\left.\mathrm{Phe}^{225}\right)(\mathrm{lb}-\mathrm{e})$. The I108A mutant confers resistance to raltitrexed and thymitaq with respective IC $_{50}$ values of 54 and 80 times greater than the wildtype enzyme. These experiments led Sapse et al. (2) to perform quantum chemical ab initio calculations on the complexes formed by the binding of thymitaq with various mutants of Ile $\mathrm{e}^{108}$.

In this study, a mutant, G52S, within the Arg ${ }^{50}$ loop of TS was examined. This mutant, obtained using EMS mutagenesis, was found to retain full catalytic activity with a reduced affinity for thymitaq and FdUMP having Ki values of 5 and 20 times greater than that of wt TS, respectively. Other mutations in 
this region, such as $\mathrm{R} 50 \mathrm{C}$, T51A, and $\mathrm{D} 49 \mathrm{~N}$, were shown to greatly affect the activity of the enzyme. The reduced affinity of the G52S mutant for thymitaq and FdUMP was postulated to result from impaired movement of the $\mathrm{Arg}^{50}$ loop due to the effect of the G52S mutation, thereby compromising the interaction of the loop with nucleotide and folate molecules (3).

Upon binding of the substrate (dUMP) to the active site of TS, $\mathrm{Arg}^{50}$ and Gly ${ }^{52}$ side-chains reorient to accommodate binding to the phosphate moiety of dUMP. This reorientation results in a slight shift $(<1 \AA)$ of the entire $\operatorname{Arg}^{50}$ loop, a step necessary to accept the incoming folate molecule. Once the ternary complex is formed a substantial conformational change in the enzyme's tertiary structure occurs. The carboxylate of the $\mathrm{C}$-terminal valine moves into the active site where it and $\mathrm{N}-1$ of $\mathrm{CH}_{2} \mathrm{H}_{4}$ folate form hydrogen-bond networks with $\mathrm{Arg}^{50}$ through fixed $\mathrm{H}_{2} \mathrm{O}$ molecules. Thus, this flexible $\mathrm{Arg}^{50}$ residue acts as a bridge linking the enzyme $\mathrm{C}$ terminus, substrate, and cofactor together and plays an important role in the enzyme's mechanism of action by binding to the phosphate group of dUMP (4).

To examine the hypothesis that the reduced affinity of G52S for Thymitaq and FdUMP is a result of impaired movement of the $\mathrm{Arg}^{50}$ loop and to investigate further the Arg-FdUMP interaction, this study applied ab initio (Hartree-Fock) and semiempirical calculations to the system.

\section{Methods and Results}

Interactions Between the Methylguanidinium Ion as a Model for the Arg ${ }^{50}$ Residue and the Phosphate Group as a Model for FdUMP in TS

The Gaussian 98 computer program (5) was used to perform ab initio calculations at HF/6-311G* (Hartree-Fock) and at MP2/6-311G* level to investigate the interaction between the methylguanidinium ion as a model for the $\mathrm{Arg}^{50}$ residue in thymidylate synthase, and a phosphate group as a model for the inhibitor FdUMP. The $6-311 G^{*}$ basis set involves one Slater orbital for the description of core electrons and three Slater orbitals for the description of valence electrons, one expanded in a series of three gaussians and the other two approximated by one gaussian each. In addition, $\mathrm{d}$ functions are set on the non-hydrogen atoms. The correlation energy is included in the calculations via the use of the Moller-Plesset perturbation theory, with the second-order term, MP2, applied to all electrons. The two systems considered are

a. Complex la-the complex formed by methylguanidine and the negative methyl phosphate ion (Fig. 1A), and

b. Complex $1 \mathrm{~b}-$ the complex formed by the methylguanidinium ion with the binegatively charged methyl phosphate ion (Fig. 1B).

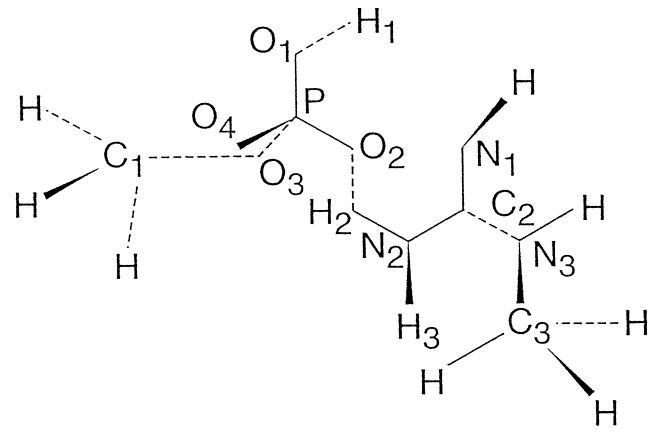

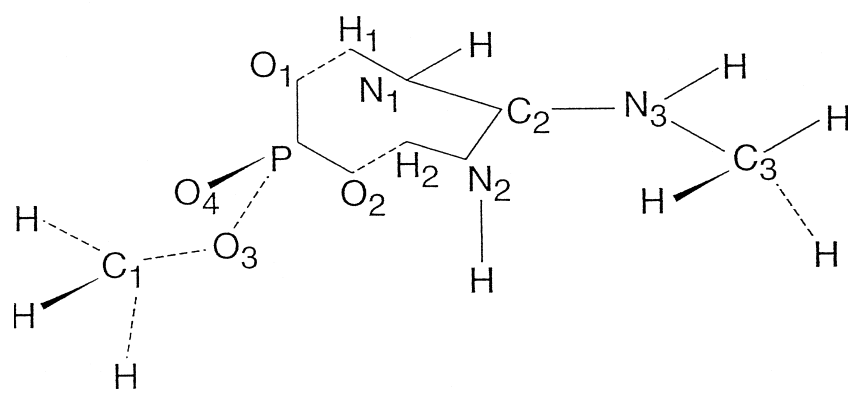

Fig. 1. (A) The complex formed by the methylguanidine and the negative methyl phosphate ion. (B) The complex formed by the methylguanidinium ion and the binegative methyl phosphate ion.

The energies of the complexes and of the subsystems, as obtained via geometry optimization, are shown in Table 1. Table 2 shows the optimized parameters of the complexes.

Comparison of the Structures of the $\mathrm{Arg}^{50}-\mathrm{Thr}^{51}-\mathrm{Gly}^{52}$ and the $\mathrm{Arg}^{50}-\mathrm{Thr}^{51}-$ Ser $^{52}$ Peptides

The blocked $\mathrm{Arg}^{50}-\mathrm{Thr}^{51}-\mathrm{Gly}^{52}$ and $\mathrm{Arg}^{50}-\mathrm{Th}^{51}$ $\mathrm{Ser}^{52}$ tripeptides are geometry optimized using the 6-31G* basis set at Hartree-Fock level, as implemented by the PC Spartan program (6).

Figure 2A shows the optimized $\mathrm{Arg}^{50}-\mathrm{Thr}^{51}-\mathrm{Gly}^{52}$ tripeptide and Figure 2B shows the Arg ${ }^{50}-\mathrm{Thr}^{51}-\mathrm{Ser}^{52}$ optimized tripeptide. Figure 2C shows the two peptides superimposed at the threonine residue.

The $\mathrm{Arg}^{50}$ loop in hTS $\left(\mathrm{Arg}^{46}-\mathrm{Lys}^{47}-\mathrm{Asp}^{48}\right.$. $\left.\mathrm{Asp}^{49}-\mathrm{Arg}^{50}-\mathrm{Thr}^{51}-\mathrm{Gly}^{52}-\mathrm{Thr}^{53}-\mathrm{Gly}^{54}-\mathrm{Thr}^{55}\right)$ was op-

Table 1. Energies of the complexes and subsystems at MP2 (full)/6-311G* computational level (au)

\begin{tabular}{ll}
\hline Complex la & -925.96762 \\
Complex lb & -925.91718 \\
Methylphosphate (negative ion) & -681.86106 \\
Methylphosphate (binegative ion) & -681.11432 \\
Methylguanidine & -244.07450 \\
Methylguanidinium ion & -244.47037
\end{tabular}


Table 2. Geometric parameters of the complexes ( $\AA$ and degrees) optimized at MP2 (full)/6-311G* level

\begin{tabular}{lcc}
\hline & \multicolumn{2}{c}{ Bond Lengths } \\
\cline { 2 - 3 } Group & Complex la & Complex 1b \\
\hline PO1 & 1.654 & 1.552 \\
PO3 & 1.503 & 1.542 \\
PO4 & 1.657 & 1.698 \\
C103 & 1.487 & 1.506 \\
$\mathrm{H} 101$ & 1.414 & 1.403 \\
$\mathrm{~N} 1 \mathrm{H} 1$ & 0.960 & 1.510 \\
$\mathrm{C} 2 \mathrm{~N} 1$ & 4.040 & $1.01^{*}$ \\
$\mathrm{C} 2 \mathrm{~N} 2$ & 1.283 & 1.322 \\
$\mathrm{C} 2 \mathrm{~N} 3$ & 1.394 & 1.318 \\
$\mathrm{C} 3 \mathrm{~N} 3$ & 1.406 & 1.370 \\
$\mathrm{O} 2 \mathrm{H} 2$ & 1.453 & 1.443 \\
$\mathrm{O} 3 \mathrm{H} 3$ & 1.875 & \\
\end{tabular}

Complex 1a

Bond Angles

\begin{tabular}{lcc} 
& Complex la & Complex lb \\
\hline O1PO2 & 107.9 & 110.6 \\
O2PO3 & 100.1 & 103.4 \\
O3PO4 & 107.3 & 115.3 \\
C103P & 116.0 & 113.8 \\
H101P & 109.0 & 122.2 \\
N1H101 & 236.0 & $180.0 *$ \\
C2N1H1 & 122.8 & 122.0 \\
N2C2N1 & 127.9 & 120.7 \\
N3C2N2 & 111.6 & 119.9 \\
C3N3C2 & 115.7 & 123.4 \\
& & \\
\hline & \multicolumn{2}{c}{ Dihedral Angles } \\
\cline { 2 - 3 } & Complex la & Complex 1b \\
\hline O3PO102 & 108.0 & 107.2 \\
C103PO2 & -137.9 & -136.2 \\
N1H10102 & 186.6 & 177.3 \\
& 0.0 & 0.0 \\
\hline
\end{tabular}

*Kept frozen.

timized with the MNDO semi-empirical method and modeled using the Insight II program on a Silicon Graphics station. The same procedure was used for the loop with Gly $^{52}$ replaced by a Ser residue. The superimposed peptides of the $\mathrm{Arg}^{50}$ loop are shown in Figure 3. Figure 4 shows the superimposition of the tripeptides $\mathrm{Arg}^{50}-\mathrm{Thr}^{51}-\mathrm{Gly}^{52}$ and $\mathrm{Arg}^{50}-\mathrm{Thr}^{51}$ $\mathrm{Ser}^{52}$ as optimized by the MNDO method and modeled by Insight II. Figure 5 shows the loop as part of rat thymidylate synthase complexed with dUMP and the anticancer drug raltitrexed (Tomudex) (7).
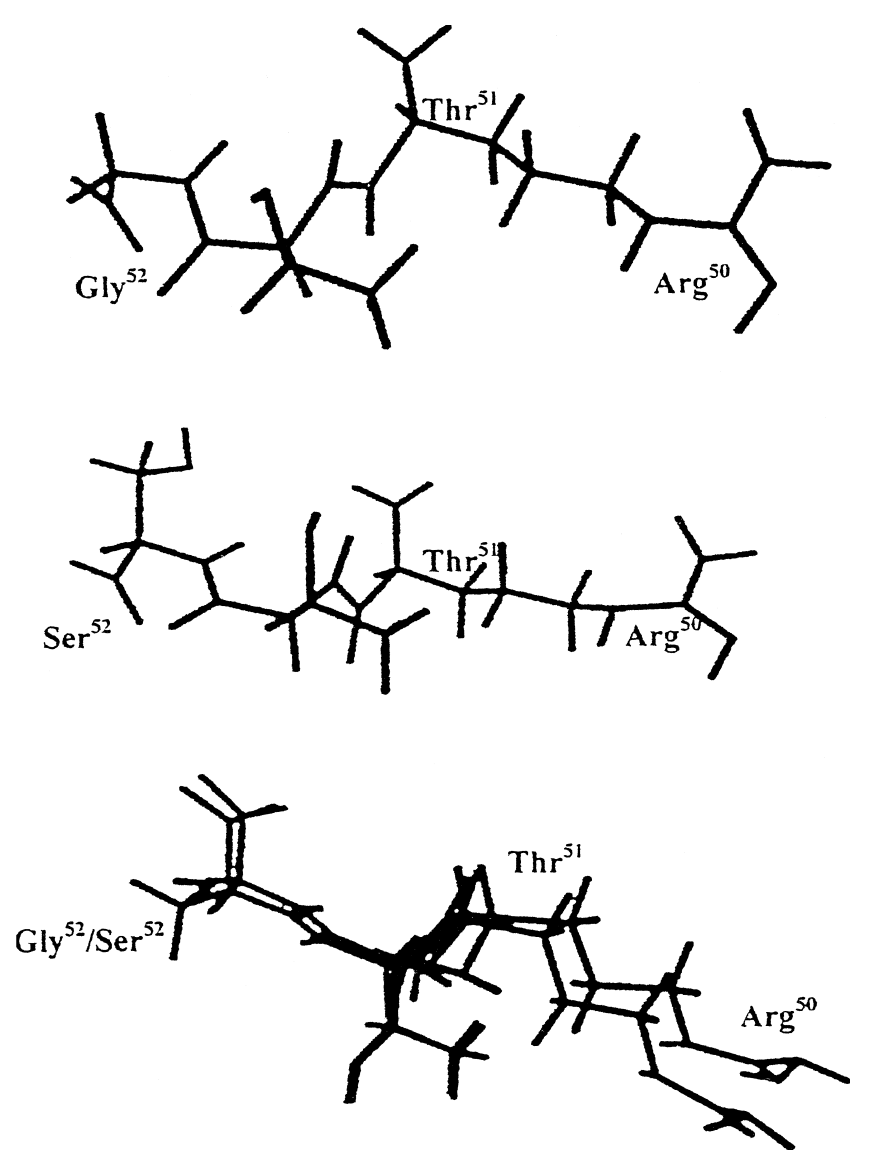

Fig. 2. Optimization of the tripeptide at HF/6-31G* level. (A) $\mathrm{Arg}^{50}-\mathrm{Thr}^{51}-\mathrm{Gly}^{52}$ optimized at HF/6-31G* level. (B) $\mathrm{Arg}^{50}$ $\mathrm{Thr}^{51}-\mathrm{Ser}^{52}$ optimized at HF/6-31G* level. (C) $\mathrm{Arg}^{50}-\mathrm{Thr}^{51}-\mathrm{Gly}^{52}$ and $\mathrm{Arg}^{50}-\mathrm{Thr}^{51}-\mathrm{Ser}^{52}$ superimposed at $\mathrm{Thr}^{51}$ residue.

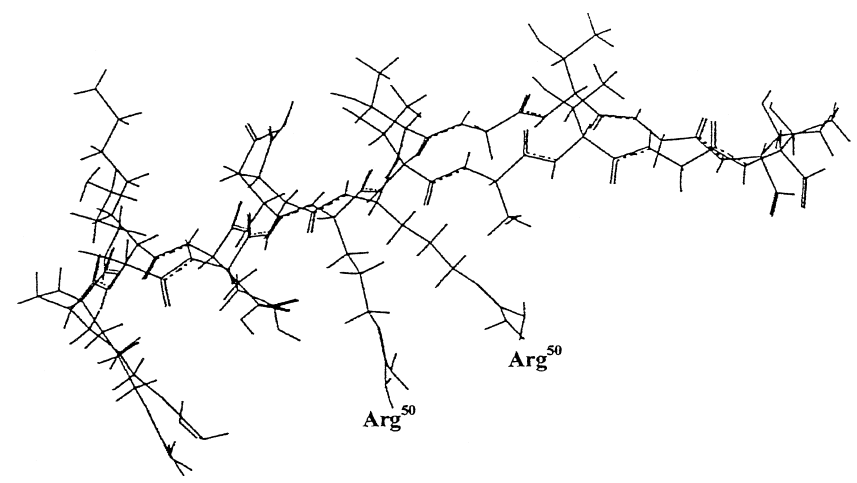

Fig. 3. The wild-type $\mathrm{Arg}^{50}$ loop and the $\mathrm{Arg}^{50}$ loop with the G52S mutation optimized at MNDO level and superimposed. 


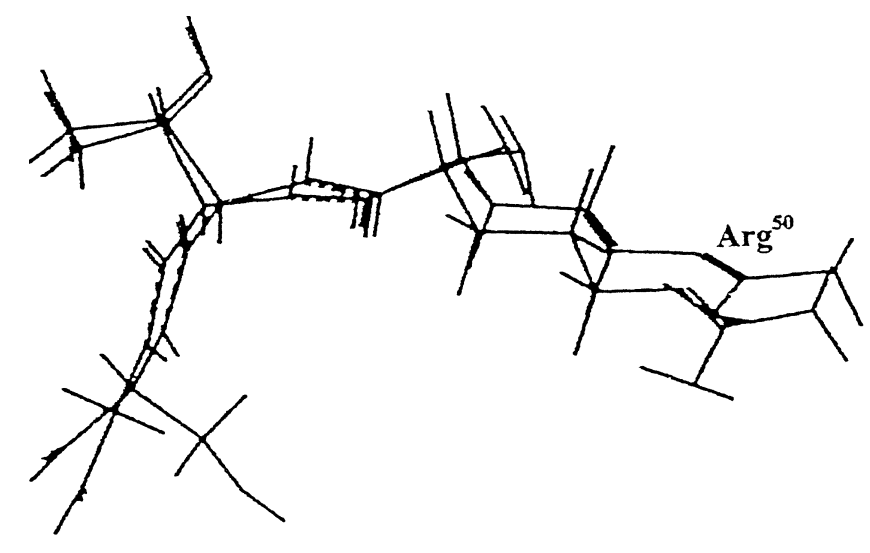

Fig. 4. $\mathrm{Arg}^{50}-\mathrm{Thr}^{51}-\mathrm{Gly}^{52}$ and $\mathrm{Arg}^{50}-\mathrm{Thr}^{51}-\mathrm{Ser}^{52}$ optimized at MNDO level and superimposed.

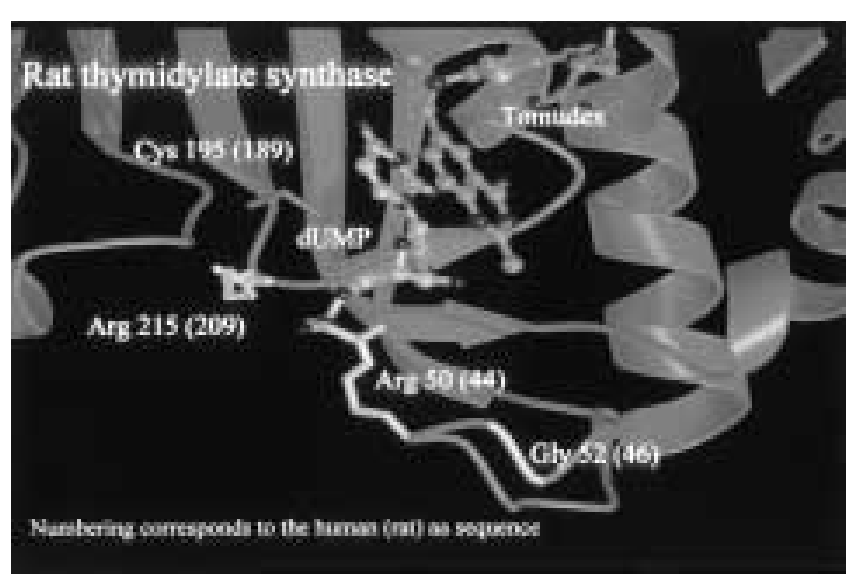

Fig. 5. The $\mathrm{Arg}^{50}$ loop as part of rat thymidylate synthase (9).

\section{Discussion}

The complex formed by the negative methylphosphate and methylmethylguanidine (Complex la; Fig. 1A) is more stable than the one formed by the methylmethylguanidinium ion and the binegative methylphosphate ion (Complex 1b; Fig. 1B), by $31.80 \mathrm{kcal} / \mathrm{mol}$ at $\mathrm{HF} / 6-311 \mathrm{G}^{*} / / \mathrm{MP} 2$ (full) $/ 6-311 \mathrm{G}^{*}$ level and by $31.64 \mathrm{kcal} / \mathrm{mol}$ at MP2 (full) $/ 6-311 \mathrm{G}^{*}$ level. This is not surprising: the proton affinity of methylguanine is only $257.38 \mathrm{kcal} / \mathrm{mol}$ at $\mathrm{HF} / 6$ $311 G^{*} / / \mathrm{MP} 2$ (full)/6-311G* level and 248.41 $\mathrm{kcal} / \mathrm{mol}$ at MP2 (full)/6-311G* level, whereas the proton affinity of the binegative methylphosphate ion is $473.29 \mathrm{kcal} / \mathrm{mol}$ at $\mathrm{HF} / 6-311 \mathrm{G}^{*} / / \mathrm{MP} 2$ (full)/ 6-311G* level and $468.58 \mathrm{kcal} / \mathrm{mol}$ at MP2 (full)/6$311 \mathrm{G}^{*}$ level. On the other hand, complex $1 \mathrm{~b}$ features a much larger binding energy due to the electrostatic attraction between opposite charges, in addition to the hydrogen binding. Indeed, the binding of complex la is $16.40 \mathrm{kcal} / \mathrm{mol}$ at $\mathrm{HG} / 6-311 \mathrm{G}^{*} / / \mathrm{MP} 2$ (full) $/ 6-311 \mathrm{G}^{*}$ level and $20.12 \mathrm{kcal} / \mathrm{mol}$ at MP2 (full)/6-311G* level, while the binding energies of complex $\mathrm{lb}$ are $200.5 \mathrm{l} \mathrm{kcal} / \mathrm{mol}$ and 208.64 $\mathrm{kcal} / \mathrm{mol}$, respectively; these energies include an electrostatic interaction between a positive and a negative charge.

Complex la was optimized by allowing the methylguanidine moiety to relax into a nonplanar conformation, similar to methylguanidine itself. Indeed, if methylguanidine is optimized using a small basis set, without energy terms, it adopts a quasi-planar geometry. However, when large basis sets such as $6-311 G^{*}$ are used, methylguanidine features the hydrogens out of the plane of the carbon and the three nitrogens. On the contrary, the guanidium ion is always planar, due to the $\mathrm{Y}$ aromaticity. As a consequence, when the methylguanidine moiety is forced to stay planar, the most stable complex features a hydrogen bond between $\mathrm{Hl}$ (placed on the oxygen $\mathrm{Ol}$ ) and the nitrogen atom $\mathrm{Nl}$, on which there is only one hydrogen. This complex has a binding energy about half of the complex la, which features one of the hydrogens set on nitrogen N2 hydrogen bound to $\mathrm{O} 2$, at a distance of $1.88 \AA$. It may be concluded that the strongest phosphatemethylguanidine interaction occurs via the hydrogen bond formed by one of the hydrogens set on a nitrogen and an oxygen of the phosphate ion. As shown before, this complex is more stable than complex $\mathrm{lb}$.

As seen from Figure 2C, the HF/6-31 ${ }^{*}$ optimization of the $\mathrm{Arg}^{50}-\mathrm{Thr}^{51}-\mathrm{Gly}^{52}$ and the $\mathrm{Arg}^{50}$ $\mathrm{Thr}^{51}-\mathrm{Ser}^{52}$ predicts a significant displacement of the $\mathrm{Arg}^{50}$ residue when Gly ${ }^{52}$ is replaced by Ser. The difference in the geometries of the two tripeptides might be attributed to electrostatic effects introduced by the presence of the electronegative oxygen present in serine.

When the whole $\mathrm{Arg}^{50}$ loop is optimized with the MNDO method, as seen in Figure 3, the displacement of $\mathrm{Arg}^{50}$ is seen even more. This displacement is also observed in Figure 4, which shows the MNDO optimization of the two tripeptides.

In conclusion, our calculations of the interaction between the methylguanidinium ion as a model for the $\mathrm{Arg}^{50}$ residue and the phosphate group as a model for FdUMP in TS predict a significant displacement of the $\mathrm{Arg}^{50}$ residue upon mutation of $\mathrm{Gly}^{52}$ to Ser. It is possible that this displacement could result in impaired movement of the entire $\mathrm{Arg}^{50}$ loop, accounting for loss of binding of FdUMP.

Because loss of binding is not seen with the natural substrate, dUMP, it is suggested that the presence of the fluorine on FdUMP may influence the flexibility of the phosphate group.

\section{References}

la. Sotelo-Mundo RR, Ciesla J, Dzik JM, et al. (1999) Crystal structures of rat thymidylate synthase inhibited by Tomudex, a potent anticancer drug. Biochemistry 38: 1087-1094.

1b. Barbour KW, Berger SH, Berger FG. (1990) Single amino acid substitution defines a naturally occurring genetic variant of human thymidylate synthase. Mol. Pharmacol. 37: 515-518. 
lc. Barbour KW, Hoganson DK, Berger SH, Berger FG. (1992) A naturally occurring tyrosine to histidine replacement at residue 33 of human thymidylate synthase confers resistance to 5-fluoro-2'-deoxyuridine in mammalian and bacterial cells. Mol. Pharmacol. 42: 242-248.

1d. Hughey CT, Barbour KW, Berger FG, Berger SH. (1993) Functional effects of a naturally occurring amino acid substitution in human thymidylate synthase. Mol. Pharmacol. 44: 316-323.

1e. Tong Y, Xinyue L-C, Ercikan-Abali EA, Zhao S-C, Banerjee D, Maley F, Bertino JR. (1998) Probing the folate-binding site of human thymidylate synthase by site-directed mutagenesis. J. Biol. Chem. 273: 31209-31214.

2. Sapse SD, Tong Y, Bertino JR, Sapse AM. (1999) Ab initio studies of some amino acid residue complexes with 4mercaptopyridine as a model for thymitaq (AG337), an inhibitor of thymidylate synthase. Cancer Invest. 17: 396401.

3. Tong Y, Xinyue L-C, Ercikan-Abali EA, Capiaux GM, Zhao S-C, Banerjee D, Bertino JR. (1998) Isolation and characterization of Thymitaq (AG337) and 5-fluoro-2-deoxyuridylateresistant mutants of human thymidylate synthase from ethyl methanesulfonate-exposed human sarcoma HT1080 cells. J. Biol. Chem. 273: 11611-11618.

4. Finer-Moore J, Fauman EB, Foster PG, Perry KM, Santi DV, Stroud RM. (1993) Refined structures of substrate-bound and phosphate-bound thymidylate synthase from Lactobacillus casei. J. Mol. Biol. 232: 1101-1116.

5. Frisch MJ, Trucks GW, Schlegel HB, et al. (1998) Gaussian 98. Gaussian Inc., Pittsburgh.

6. Deppmeier B, Driesser AJ, et al. Spartan PC. Irvine, CA, Wavefunction Inc. 